2006-671: STRUCTURED APPROACH IN TEACHING INTERMEDIATE MECHANICS OF MATERIALS

Madhukar Vable, Michigan Technological University 


\section{Structured approach in teaching intermediate mechanics of materials}

\section{Abstract}

A pedagogy is presented for use in second course of mechanics of materials in which material and geometric complexities can be added in a very compact manner to the basic theories of axial members, circular shafts and bending of beams that the students have learnt in their introductory course of mechanics of materials. The pedagogy uses the same process of adding complexities many times, thus improving the chances of retention and understanding of the principles, patterns, and logic structure intrinsic to mechanics of materials. Students performance and feedback indicates that the concepts of the introductory course are consolidated and the students develop an appreciation of vast array of complexities and how these can be added to the theories of onedimensional structural elements.

\section{Introduction}

Plastics engineering is becoming a popular new minor, bio-medical engineering is a growing new discipline - both require understanding of the mechanics of inelastic and non-linear material behavior in their applications of stress analysis. Applications of inelastic and non-linear material is also growing as metals compete with new materials in engineering design by operating in the plastic region through pre-stressing. The growing usage of metal matrix composites, polymer composites, reinforced concrete, wooden beams stiffened with steel strips and other laminated structures are emphasizing the need to expose students to analysis of one-dimensional composite structural members. The ubiquity of finite element method in engineering analysis and design not only emphasizes the educational importance of energy principles and the concepts of finite element method, but as significantly requires the understanding of stress and strain transformation in three dimensions and failure theories in order to evaluate and use the results produced by commercial finite element computer software. The capstone senior design projects have added to the importance of understanding unsymmetric bending and the concept and use of shear centers in design. Beam and shaft vibrations, beams on elastic foundations, Timoshenko beam, etc. are among the many topics in existing aerospace, civil, and mechanical engineering courses that use the principles of mechanics of materials. If a student is to be taught the mechanics of the topics described and not be overwhelmed with all the complexities inherent in these topics, then the presentation of the material must have coherence and compactness that consolidates what the students have already learned in the introductory course and builds on it.

The above paragraph emphasizes the need for a second course in mechanics of materials "Intermediate mechanics of materials" and the importance of development of educational material that highlights the principles, patterns and logic structure intrinsic in mechanics. Several questions arise:

1. Is there room in today's engineering curriculum for a second course in mechanics of material?

2. What should be the course content for intermediate mechanics of material?

3. What books are available for teaching of the course? 
4. What is meant by structured approach in teaching intermediate mechanics of materials?

Answers to the above questions are elaborated in this paper.

\section{Room for a second course in engineering curriculum}

Why is the introductory course of mechanics of materials a required course in most engineering disciplines? The educational philosophy behind the requirement is to teach common mechanics concepts and principles in a single course and teach the extensions and applications of the mechanics concepts and principles in individual disciplines. This educational philosophy addresses the need of interdisciplinary education while realizing curriculum efficiency by reducing duplication of course content - an important consideration as educators attempt to modernize engineering education by incorporating research into a burgeoning curriculum. The introductory course of mechanics of materials has served the engineering community well.

The intermediate mechanics of materials course in a manner similar to the introductory course can be used to teach the common stress analysis principles used in plastic engineering, bio-medical engineering, area of composites, thin-walled structure analysis and design used in aerospace, civil, and mechanical engineering, and topics that will help students interpret results from finite element method that is now used in most disciplines for stress analysis and design. The intermediate mechanics of materials course could be an elective course providing service to the new disciplines and modern topics without adding pressure to traditional curriculums.

\section{Books and Course Content}

Intermediate mechanics of materials or advanced mechanics of materials are usually the second and / or third course in mechanics of materials when they exist in engineering curriculum. The publisher Oxford University Press market survey shows that approximately $40 \%$ of engineering schools have a second course in mechanics of materials. The intermediate mechanics of materials is usually designed for juniors / seniors and open to graduate students. The advanced mechanics of materials is usually designed for graduate students and open to seniors. There is some commonality between the two courses but no consensus on the topics and the depth of coverage of a topic as became clear from the reviewers comments on the forthcoming book ${ }^{1}$ by the author. The books for the two courses named by the reviewers are by Barber ${ }^{2}$, Borsei, Schmidt, and Sidebottom $^{3}$, Budynas ${ }^{4}$, Cook and Young ${ }^{5}$, Gere $^{6}$, Shames and Cozzarelli ${ }^{7}$, Solecki and Conant ${ }^{8}$, and Ugural and Fenster ${ }^{9}$. Currently no single textbook so far cover all topics of instructor's interest. More than one book is often used in a course and instructors often supplement the books with their own notes. Thus, as things stand, the instructor teaching the intermediate mechanics of materials course can use the optional topics from a textbook designed primarily to teach the introductory course or using selected topics from a book primarily designed for graduate course on advanced mechanics of materials and supplement the book(s) with ones own notes. The pedagogical needs of the students with respect to the theoretical details, numerical examples, and post-text problems are difficult to meet and put undue burden on the instructor teaching the course-such has been mine and my colleagues experience in teaching this course in the past twenty years and echoed by the reviewers who teach intermediate mechanics of materials course. 
Noting that the intermediate mechanics of materials course is primarily geared towards undergraduate juniors and senior students, the author believes, with the exception of few topics the course should be primarily geared to one-dimensional structural elements with material and geometric complexities. This belief is based on the following observations:

1. Most of the applications in bio-medical engineering, plastic engineering, thinwalled structural members in aerospace, civil, and mechanical engineering, dynamics and vibration courses, that an undergraduate student will see are predominantly one-dimensional structural members.

2. In most curriculums undergraduate juniors / seniors have not been exposed to enough mathematics that is needed for advanced mechanics of material topics.

3. There is a need to reinforce the concepts learned in the introductory mechanics of materials course.

Described below are the major topics that could be taught in an intermediate mechanics of materials course. The reader can see more details of the topics from the author's forthcoming book on his web page: http://www.me.mtu.edu/\%7Emavable/. The reader can also obtain lecture slides for use in their own lecture on this web page. The slides are in pdf format that can be easily downloaded and can be used in courses other than intermediate mechanics of materials.

\section{List of topics}

1. Stress and strain: Transformation in two and three dimensions by matrix method; Finite difference approximation of strain

2. Linear material models: Monoclinic, Orthotropic, Transversely isotropic, Isotropic material.

3. Failure and design: Failure theories; Stress concentration factor; Stress intensity factor; Fatigue.

4. Basic structural members: Logic in structural analysis; Basic theories of one dimensional structural members; Boundary value problems using discontinuity functions; Symmetric bending of curved beams.

5. Composite structural members: Axial, Circular Shafts, and Symmetric beams; Introduction to micro mechanics of composites

6. Inelastic structural behavior: Initial stress or strain, Temperature, and Viscoelasticity in axial members. Elastic-perfectly plastic and power law material models in axial, circular shafts, and symmetric bending of beams.

7. Thin walled structural members: Unsymmetric bending of beams, Shear stress in and shear centers of open and closed sections; Torsion of multi-cell tubes; Combined loading.

8. Energy methods: Strain energy; Linear Strain Energy Density; Work; Virtual Work; Dummy unit load method; Castigliano's theorems; Deflection of curved beams; Minimum Potential Energy; Rayleigh-Ritz method.

9. Elasticity and mechanics of materials: Elasticity equations; Thick Hollow Cylinders; Thin disk; Airy stress function; Solution by polynomials; Torsion of non-circular shafts.

10. Finite element method: Terminology; Lagrange polynomials; Axial elements; Circular shaft elements; Symmetric beam elements; Finite element equations in 2-dimensions.

The above list contains more subject material then can be covered in a three credit semester course because it caters to varied interests of instructors of an elective course. The mathematics required is elementary knowledge of partial derivatives, some matrix algebra and simple calculus 
which most undergraduate students have been exposed in their curriculum. Though the mathematics is not involved, the algebra can be tedious. Upper end calculators and symbolically manipulators such as Mathematica, Maple, or Matlab can reduce the tedium, but each instructor will have to define what is permissible.

As mentioned earlier the focus of the proposed course is on one-dimensional structural members with a few exceptions. These few exceptions that are in the above list are discussed briefly in this paragraph. In stress and strain transformation the familiar equations that the student saw in their introductory course can be cast in a matrix form and principal stresses / strains and directions obtained as eigenvalues and eigenvectors. Once the matrix algebra of stress transformation is explained in two-dimension, it can then be extended to three-dimensions. In material models, in addition to exposing the students to the four models in topic two of the list above, it can be shown through numerical examples that the principal directions for stresses and strains are only same for isotropic material and not same for other models such as orthotropic materials. Failure theories and stress intensity factor for mode one use the calculations of principal stresses. The stress intensity factor can be introduced like the stress concentration factor for use in strength based analysis and design. The topic of elasticity can be introduced so that its relationship to mechanics of materials elaborated and used to systematically obtain results for thick cylinders, thin rotating discs and torsion of non-circular shafts, all within the capability of an undergraduate student.

The next section discusses the presentation of subject matter for one-dimensional structural members with material and geometric complexities that are described in topics 4 through 7 in the list above. Topics 8 and 10 on 'energy methods' and 'finite element method' can be elaborated using one-dimensional structural members.

\section{Structured approach to intermediate mechanics of materials}

The pedagogy being proposed by the author is to show the students the logic used in mechanics of materials and by repetitive application of the derivation procedure show how material and geometric complexities can be incorporated into the basic elementary theory of one-dimensional structural members.

The logic symbolically shown in Fig. 1 is used in mechanics of materials for developing simple theories discussed in the introductory course of mechanics of materials to the advanced theories in graduate courses. It is possible to start at any point in the logic and move either in clock-wise direction shown by the filled arrows $\rightarrow$ or in counter-clockwise direction shown by the hollow arrows $\rightarrow$. It is not possible to relate displacement directly to external forces without imposing limitation and making assumptions regarding the geometry of the body, material behavior, and external loading. This is emphasized by the absence of arrows directly linking displacement and external forces in Fig. 1.

In the introductory course of mechanics of materials the students learn the theories for axial rods, torsion of circular shaft, and symmetric bending of beams. The derivation of all three theories is presented in a consolidated form as a synopsis in Table 1 which highlights the commonality in the three theories and the modular character that is depicted in Fig.1. The variables in Table 1 are as follows: 
$\mathrm{u}, \mathrm{v}$, and $\mathrm{w}$ are the displacements in $\mathrm{x}, \mathrm{y}$, and $\mathrm{z}$ direction respectively.

$\phi$ is the rotation of the cross-section, considered positive counter-clockwise with respect to the $x$-axis. $\mathrm{p}_{\mathrm{x}}(\mathrm{x})$ and $\mathrm{p}_{\mathrm{y}}(\mathrm{x})$ are the external distributed force per unit length in the $\mathrm{x}$ and $\mathrm{y}$ direction, respectively.

$t(x)$ is the external distributed torque per unit length, considered positive counter-clockwise with respect to the $\mathrm{x}$-axis.

$\boldsymbol{N}, \boldsymbol{V}_{\mathrm{y}}$, and $\boldsymbol{V}_{\mathrm{z}}$ are the internal axial and internal shear forces.

$\boldsymbol{T}, \boldsymbol{M}_{\mathrm{y}}$, and $\boldsymbol{M}_{\mathrm{z}}$ are the internal torque and internal bending moments.

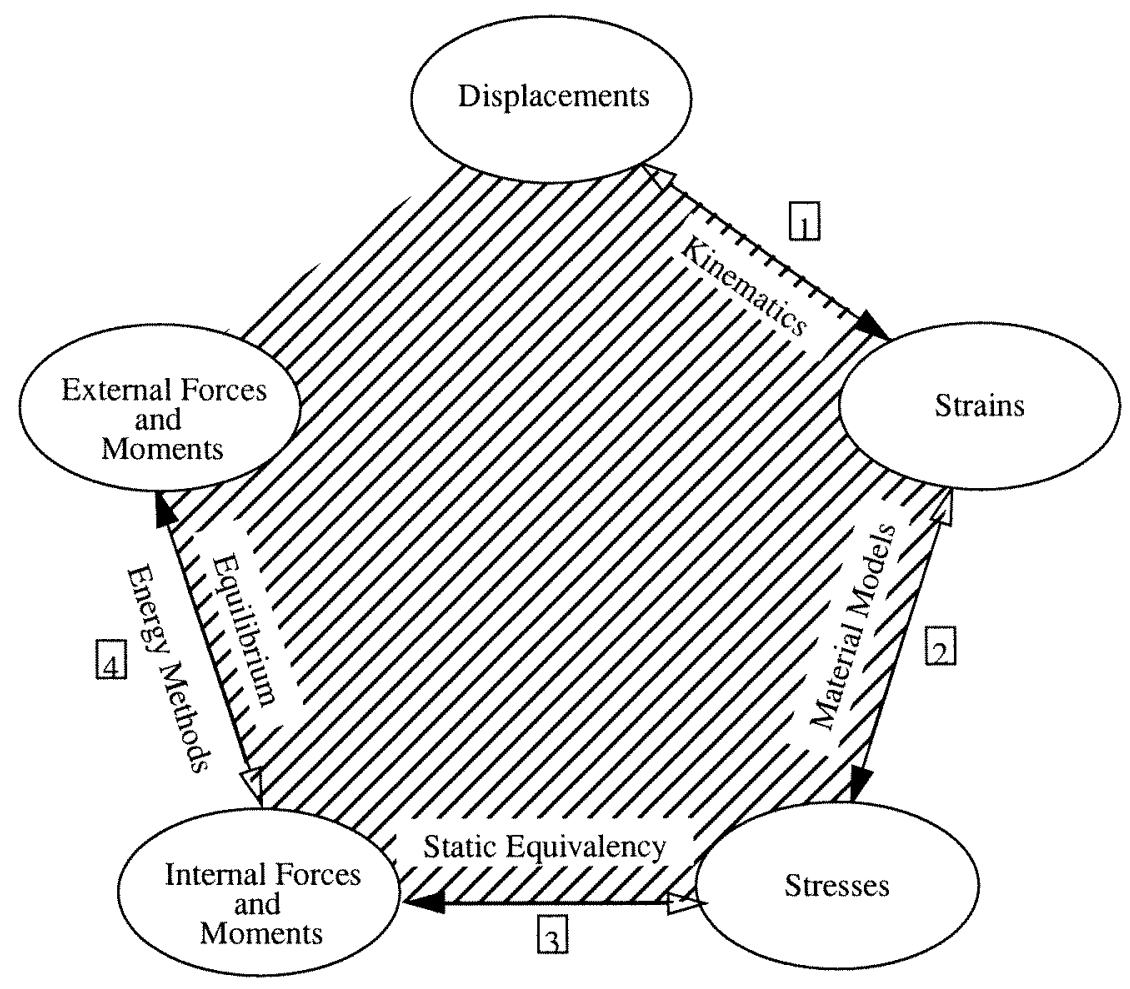

Fig. 1. Logic in structural analysis.

The four links connecting the five variables shown in Fig. 1 are: kinematic equations between displacements and strains; the constitutive equations between strains and stresses; the equivalency equations between stresses and internal forces; and the equilibrium equations between internal and external forces. It is emphasized that if the assumptions in one module are changed then these changes only affect the equations in that module while the equations in other modules remain unchanged. With this view consider the following list of added complexities to the basic theory that the student have learned in the introductory course and shown in Table 1. The author solves some of these in class, some in his notes that will soon be a textbook, assigns some to the students as homework problems, and use some as bonus problems in exams to challenge the brighter students.

1. Dynamic problems

The primary change is in the free body diagrams of the differential element which now include 
dynamic terms as shown in Fig. 2., where $\rho_{m}$ is the mass density. One of the problems can be used to show the inclusion of the dynamic term and others can be assigned as homework
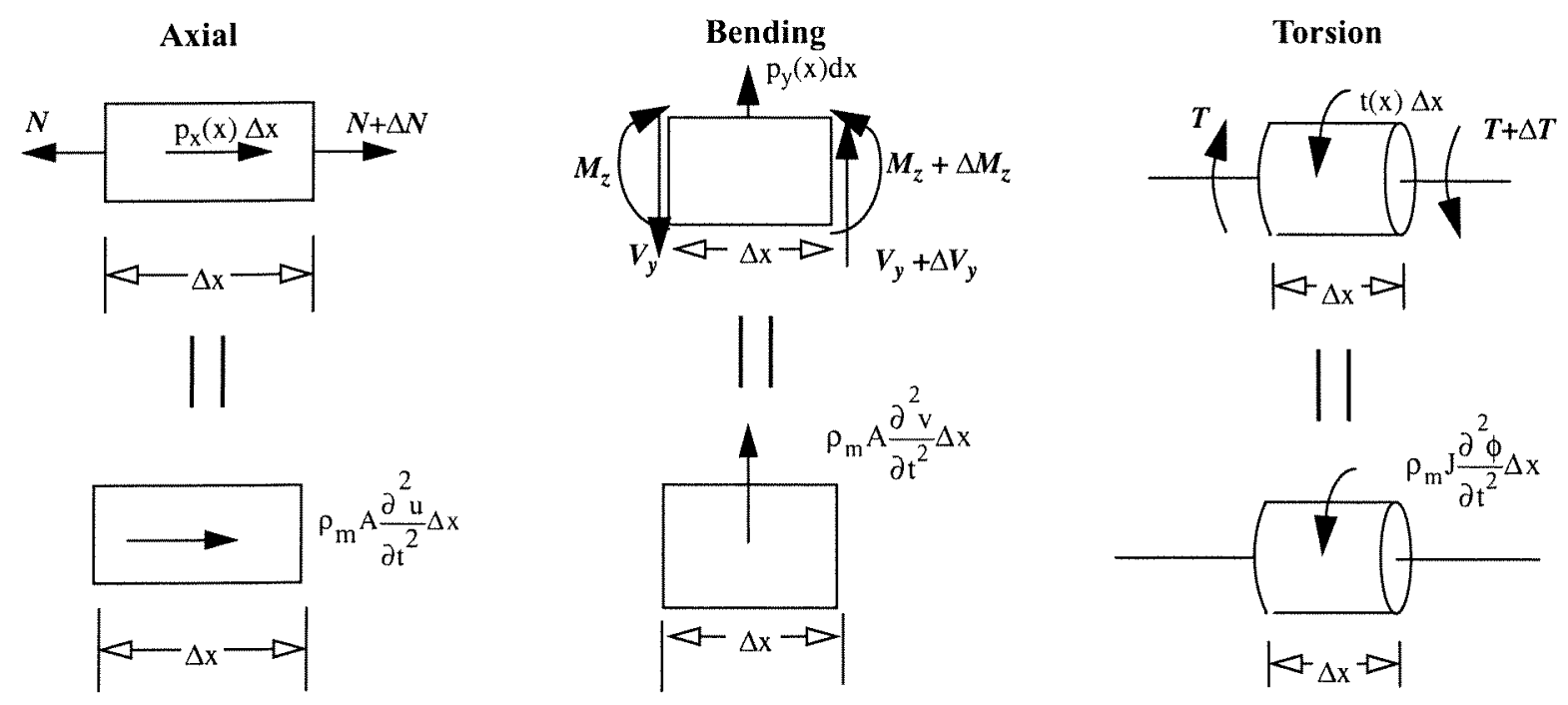

Fig. 2. Incorporation of dynamic terms.

\section{Beams on Elastic Foundations}

Once more the primary change is in the free body diagrams of the differential element which now include the foundation effect as shown in Fig.3, where, $\mathrm{k}$ is foundation modulus, i.e.,spring constant per unit length.Obtaining the differential equation with foundation effects is well within the capabilities of the students.

Fig. 3. Foundation effect

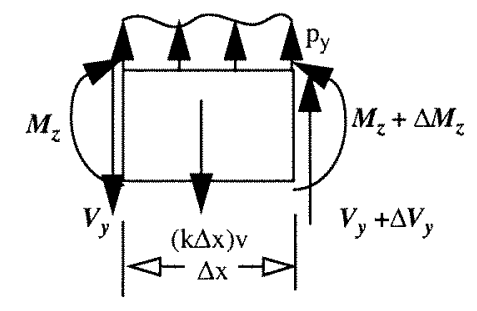

\section{Timoshenko Beams}

This problem is solved as an example problem to once more show the logic and the process of derivation. From point of view of derivation the primary change is in the kinematics. The problem is stated as follows:

In Timoshenko beams the assumption of planes remaining perpendicular to the axis of the beam is dropped to account for shear. The derivation starts with the following displacement field

$$
\mathrm{u}=-\mathrm{y} \psi(\mathrm{x}) \quad \mathrm{v}=\mathrm{v}(\mathrm{x})
$$

where, $\psi$ is the angle of rotation of the plane of cross-section from the vertical. Obtain the simplest differential equations on $v$ and $\psi$ for Timoshenko beams. 


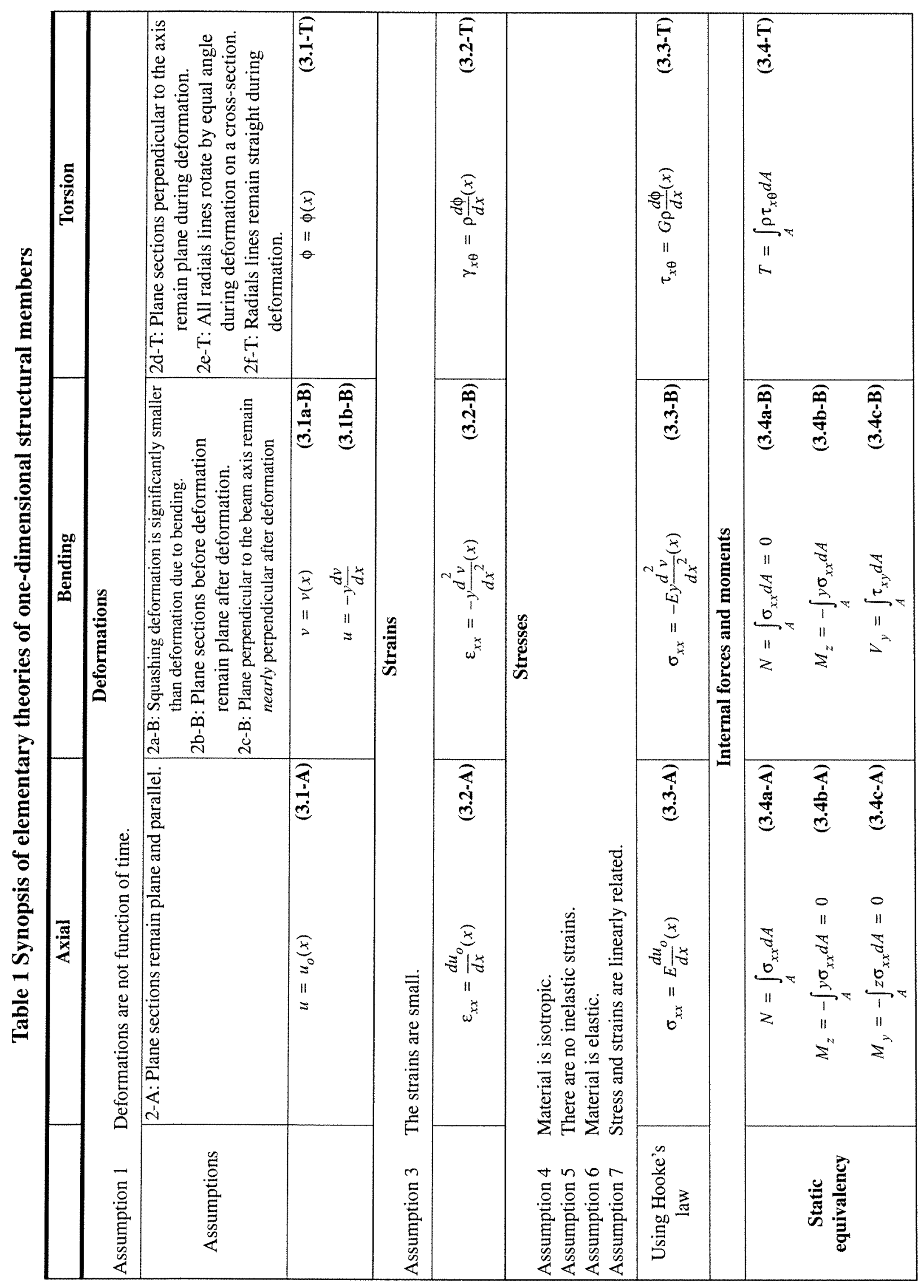




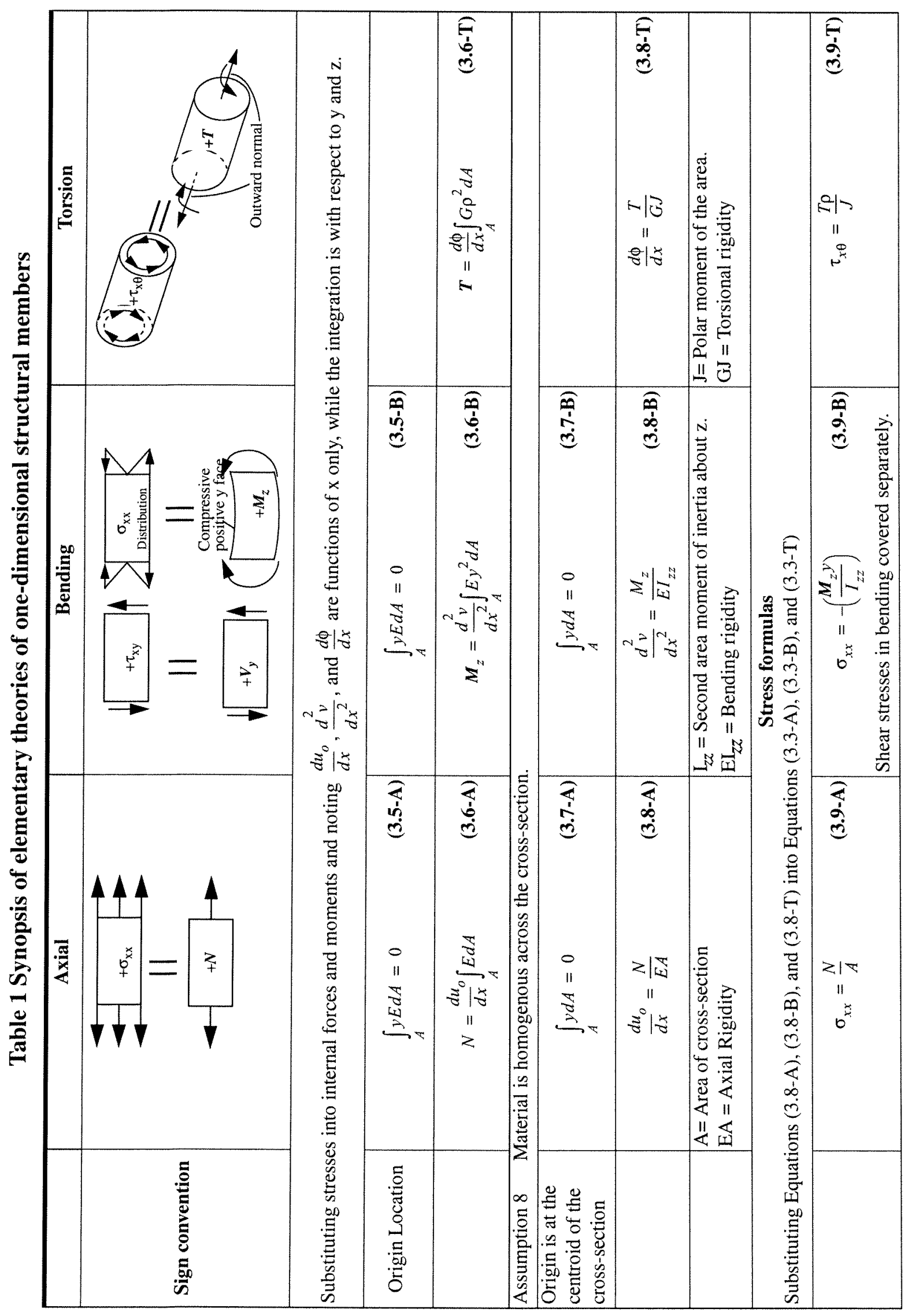




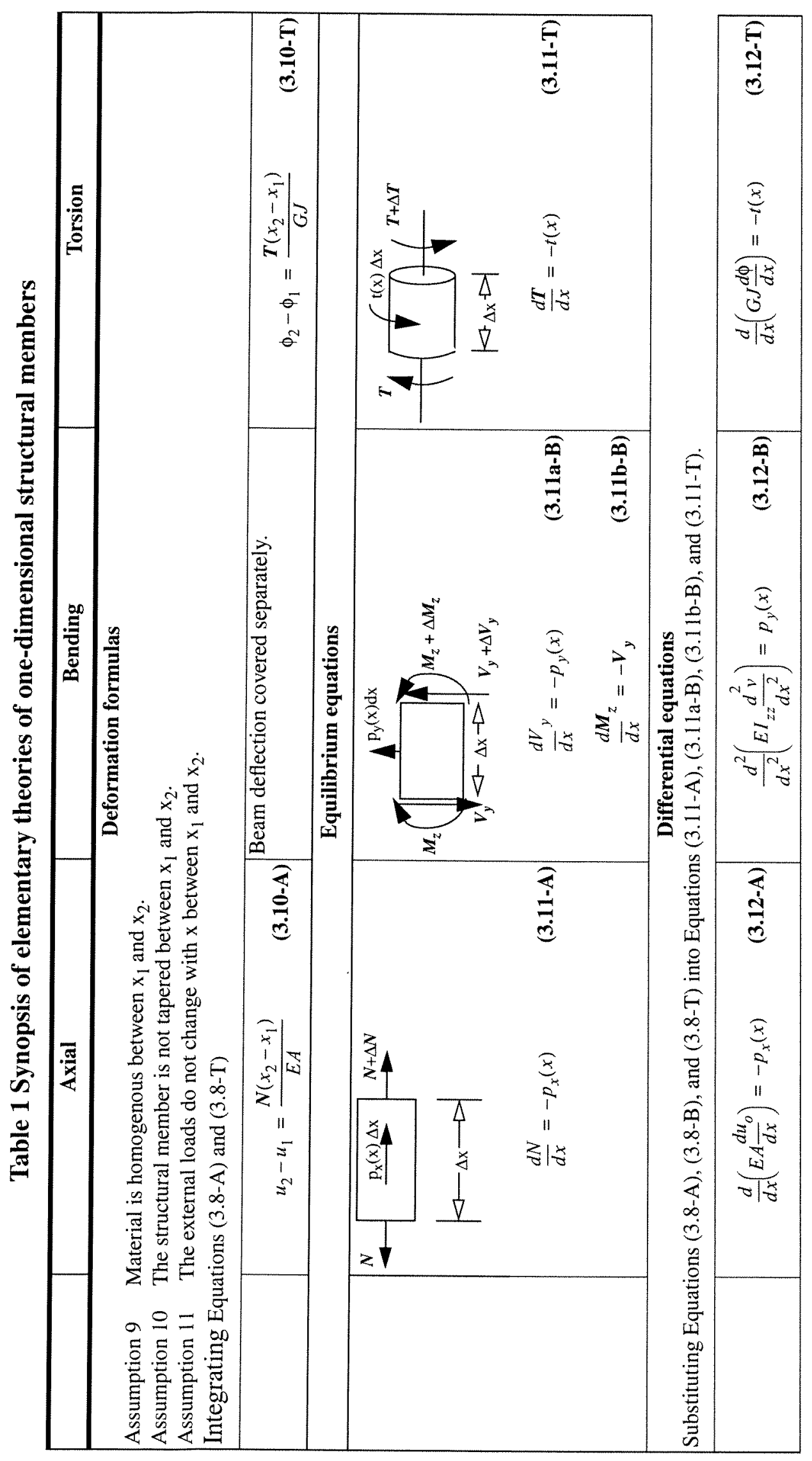

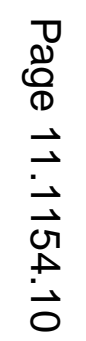




\section{Composite structural members}

The primary change is in the assumption of material homogeneity i.e., Assumption 8 in Table 1.Thus, all equations before this assumption are valid and the integral over the cross-sectional area is written as sum of the integral over each material area and the three Equations (3.6-A), (3.6B), and (3.6-T) as shown below.

$$
\begin{aligned}
& \text { Axial: } \boldsymbol{N}=\frac{d \mathrm{u}}{d \mathrm{x}} \int_{\mathrm{A}} \mathrm{EdA}=\frac{d \mathrm{u}}{d \mathrm{x}}\left[\int_{\mathrm{A}_{1}} \mathrm{E}_{1} \mathrm{dA}+\int_{\mathrm{A}_{2}} \mathrm{E}_{2} \mathrm{dA}+\cdot++\int_{\mathrm{A}_{\mathrm{n}}} \mathrm{E}_{\mathrm{n}} \mathrm{dA}\right]=\frac{d \mathrm{u}}{d \mathrm{x}}\left[\sum_{\mathrm{j}=1}^{\mathrm{n}} \mathrm{E}_{\mathrm{j}} \mathrm{A}_{\mathrm{j}}\right] \\
& \text { Bending: } \boldsymbol{M}_{\mathrm{z}}=\frac{d^{2} \mathrm{v}}{d \mathrm{x}^{2}}\left[\int_{\mathrm{A}_{1}} \mathrm{E}_{1} \mathrm{y}^{2} \mathrm{dA}+\int_{\mathrm{A}_{2}} \mathrm{E}_{2} \mathrm{y}^{2} \mathrm{dA}+\cdot++\int_{\mathrm{A}_{\mathrm{n}}} \mathrm{E}_{\mathrm{n}} \mathrm{y}^{2} \mathrm{dA}\right]=\frac{d^{2} \mathrm{v}}{d \mathrm{x}^{2}}\left[\sum_{\mathrm{j}=1}^{\mathrm{n}} \mathrm{E}_{\mathrm{j}}\left(\mathrm{I}_{\mathrm{zz}}\right)_{\mathrm{j}}\right] \\
& \text { Torsion: } \boldsymbol{T}=\frac{d \phi}{d \mathrm{x}}\left[\int_{\mathrm{A}_{1}} \mathrm{G}_{1} \rho^{2} \mathrm{dA}+\int_{\mathrm{A}_{2}} \mathrm{G}_{2} \rho^{2} \mathrm{dA}+\cdot++\int_{\mathrm{A}_{\mathrm{n}}} \mathrm{G}_{\mathrm{n}} \rho^{2} \mathrm{dA}\right]=\frac{d \phi}{d \mathrm{x}}\left[\sum_{\mathrm{j}=1}^{\mathrm{n}} \mathrm{G}_{\mathrm{j}} \mathrm{J}_{\mathrm{j}}\right]
\end{aligned}
$$

The simple modification in the derivation is repeated three times in obtaining formulas for composite axial members, shafts and beams. Since equilibrium equations are unaffected, all the tools of finding internal forces and moments such as drawing free body diagrams, axial, torque, shearmoment diagrams, discontinuity functions can all be used for solving statically determinate and indeterminate problems. Problems of wooden beams stiffened with steel strips, reinforced concrete columns, light weight laminated shafts etc. can be used to demonstrate the practical application of new formulas.

\section{Inelastic structural behavior}

The primary change is in the Hooke's law. For incorporation of initial strain or temperature change, the Hooke's law gets replaced by $\sigma_{x x}=E\left(\varepsilon_{x x}-\varepsilon_{0}\right)$, where $\varepsilon_{0}$ is the initial strain or $\varepsilon_{0}=\alpha \Delta \mathrm{T}$, where $\alpha$ is coefficient of thermal expansion and $\Delta \mathrm{T}$ is the change in temperature. Equations (3.3-A) and (3.3-B) gets replaced by the following and derivation then proceeds as before.

$$
\begin{gathered}
\text { Axial: } \sigma_{\mathrm{x} x}=\mathrm{E}\left[\frac{d \mathrm{u}_{\mathrm{o}}}{d \mathrm{x}}(\mathrm{x})-\varepsilon_{\mathrm{o}}\right] \\
\text { Bending: } \sigma_{\mathrm{xx}}=\mathrm{E}\left[-\mathrm{y} \frac{d^{2} \mathrm{v}}{d \mathrm{x}^{2}}(\mathrm{x})-\varepsilon_{\mathrm{o}}\right]
\end{gathered}
$$

For power law non-linearity the Hooke's law gets replaced by $\sigma_{\mathrm{xx}}=\mathrm{E} \varepsilon_{\mathrm{xx}}^{\mathrm{n}}$ and $\tau_{\mathrm{x} \theta}=\mathrm{G} \gamma_{\mathrm{x} \theta}^{\mathrm{n}}$, where $\mathrm{n}$ is the strain hardening index. As kinematics, static equivalency, and equilibrium equations are unchanged, new formulas can be derived showing students once more the flow of logic and derivation that is seen in Fig.1 and Table 1. 
For elastic-perfectly plastic material models and viscoelastic material models no new formulas are necessary, only a demonstration and emphasis on the use of kinematic equations, static equivalency and equilibrium equations that did not change.

\section{Unsymmetric bending of beams}

From the perspective of derivation, the primary difference between symmetric and unsymmetric bending occurs in the kinematics. Equation (3.1b-B) in Table 1 gets replaced by the one below:

$$
\mathrm{u}=-\mathrm{y} \frac{d \mathrm{v}}{d \mathrm{x}}-\mathrm{z} \frac{d \mathrm{w}}{d \mathrm{x}}
$$

The derivation process and the set of assumptions that are made at each step are the same as in Table 1 and this is once more shown to the student in obtaining the formulas for unsymmetric bending of beams. The students by now have seen the derivation process many times and can appreciate how temperature effects, material non-homogeneity can be incorporated and such problems can be used to challenge the brighter students. The author gives such problems as bonus problems in exam and there are students who can get started correctly and every once in a while there is a student who gets the problem completely right!

I believe, student performance and feed back bolster this belief, this presentation consolidates what the student learned in the introductory course, the repetitive and the compact character of derivation helps in the understanding and retention of the key ideas, and exposes the students to a vast array of complexities in the derivation of theories of one-dimensional structural elements. It is not my intension to convey the impression that students understand all the implications of all the complexities they see in this book. For greater understanding of complexities, time has to be spend with the application of the solution to the equations as is done in the courses that will use these theories, but I believe the students do learn to appreciate the mechanics of incorporating complexities into the elementary theory of one-dimensional structures they learned in the introductory course of mechanics of materials.

\section{Conclusions}

The need for a second course in mechanics of materials will continue to grow as the applications of mechanics of materials continue to grow in new and old engineering disciplines. Intermediate mechanics of material course can be a valuable service course providing students with the understanding of fundamental principles for the growing applications. This paper has presented a pedagogy which shows how material and geometric complexities can be added to the basic theories of

axial members, circular shaft and bending of beams in a very compact form. By repetitively using the same process we improve the chances of retention and understanding of the mechanics principles by the students.

\section{References}

1. Vable, M (2007) "Intermediate mechanics of materials", Oxford University Press.

2. Barber J.R., (2000) "Intermediate mechanics of materials", McGraw-Hill. 
3. Boresi, A. P., Schmidt, R. J., Sidebottom, O. M., (1993) "Advanced mechanics of materials", Fifth editions, John Wiley and Sons, Inc.

4. Budynas, R. G., (1977) "Advanced strength and applied stress analysis", McGraw-Hill.

5. Cook and Young (1999) "Advanced mechanics of materials", Prentice-Hall.

6. Gere, J.M., (2004) "Mechanics of Materials", Sixth Edition, Thomson Brooks/Cole.

7. Shames, I.H. and Cozzarelli, F.A. (1997) "Elastic and inelastic stress analysis", Taylor \& Francis.

8. Solecki R. and Conant R.J., (2003) “Advanced Mechanics of Materials", Oxford University Press.

9. Ugural, A.C. and Fenster S.K., (1995) "Advanced strength and applied elasticity", Prentice Hall Inc. 